\title{
Invaginación intestinal ileoileal causada por divertículo de Meckel
}

\author{
PATRICIO IBÁÑEZ G. ${ }^{1}$, JAIME CRUZ F. $^{2}$, LORENZA ELIZALDE R. $^{2}$, DANIELA TAPIA D. ${ }^{2}$ \\ 1. Hospital Regional de Talca. Facultad de Medicina Universidad Católica del Maule, Talca. \\ 2. Interno(a) Facultad de Medicina Universidad Católica del Maule, Talca.
}

\begin{abstract}
\section{Ileoileal intussusception due to Meckel's diverticulum}

Introduction: Intussusception is the most common cause of intestinal obstruction in infants. At this age, most cases are idiopathic and involve ileocecal locations; meanwhile ileoileal invaginations caused by an anatomic abnormality of the gastrointestinal tract, such as Meckel's diverticulum, are rare. Objective: To report two cases of infant ileoileal intussusception due to Meckel's diverticulum. Case reports: The first patient was a 6 month-old female infant who presented colicky abdominal pain associated with vomiting and bloody stools. The second patient, a 23 month-old male infant with a history of Tetralogy of Fallot and corrective surgery, and DiGeorge syndrome, who consulted the specialists for health worsened due to vomits and severe dehydration, presenting the following days, semi-liquid and non-bloody stools. Conclusions: Ileoileal intussusception is rare in infants, especially those caused by anatomical changes such as Meckel's diverticulum. This association has no pathognomonic signs and its study does not differ from the usual study of intestinal intussusception, but it is important to consider it for management purposes as it involves surgical intervention and considering that the pneumatic reduction is contraindicated.
\end{abstract}

(Key words: Intussusception, Meckel's diverticulum, treatment).

Rev Chil Pediatr 2013; 84 (2): 189-193

\section{RESUMEN}

Introducción: La invaginación intestinal constituye la principal causa de obstrucción intestinal en lactantes. En este rango etáreo, la mayoría de los casos son idiopáticos y de localización ileocecal, siendo infrecuentes las invaginaciones ileoileales originadas por una alteración anatómica del tracto gastrointestinal como el Divertículo de Meckel. Objetivo: Presentar 2 casos de invaginación intestinal ileoileal en lactantes causadas por Divertículo de Meckel. Casos clínicos: El primer caso corresponde a una lactante de sexo femenino de 6 meses que presentó cuadro de dolor abdominal cólico asociado a vómitos y deposiciones sanguinolentas. El segundo, a un lactante de sexo masculino de 1 año y 11 meses con antecedentes de Tetralogía de Fallot operada y Síndrome de Di George que consultó por compromiso del estado general, vómitos y deshidratación severa, asociándose en los días siguientes deposiciones semilíquidas no sanguinolentas. Conclusiones: La

Recibido el 08 de abril de 2012, devuelto para corregir el 04 de junio de 2012, segunda versión el 31 de julio de 2012, tercera versión el 06 de marzo de 2013, aceptado para publicación el 19 de marzo de 2013.

Este trabajo cumple con los requisitos sobre consentimiento /asentimiento informado, comité de ética, financiamiento, estudios animales y sobre la ausencia de conflictos de intereses según corresponda.

Correspondencia a:

Jaime Cruz F.

E-mail: jcruzfuentes@gmail.com 
invaginación intestinal ileoileal es infrecuente, en especial las causadas por alteraciones anatómicas como el Divertículo de Meckel en lactantes. Esta asociación no tiene signos patognomónicos y su estudio no difiere del habitual de intususcepción intestinal, pero es importante considerarla para el manejo, pues posee indicación quirúrgica y está contraindicada la reducción neumática.

(Palabras clave: Invaginación intestinal, divertículo de Meckel, tratamiento).

Rev Chil Pediatr 2013; 84 (2): 189-193

\section{Introducción}

La invaginación intestinal es una enfermedad grave que constituye la principal causa de obstrucción intestinal en niños menores de 2 años ${ }^{1}$. Se produce cuando una porción del tubo digestivo se introduce dentro de un segmento inmediatamente distal a él, a menudo cercano a la válvula ileocecal, arrastrando mesenterio y obstruyendo el retorno venoso. Esto provoca edema, congestión de la mucosa e incluso necrosis de la pared intestinal ${ }^{2}$.

La invaginación intestinal ocurre con mayor frecuencia en menores de un año ${ }^{2-5}$ con un peak entre los 3 y 9 meses de vida, incluso puede presentarse en la etapa intrauterina. Afecta con más frecuencia al sexo masculino $60-70 \%{ }^{6}$. Suele producirse en la región ileocólica; menos frecuentemente son cecocólicas y raramente, ileales.

La intususcepción se presenta típicamente en el lactante como un cuadro de inicio súbito, con dolor abdominal tipo cólico y crisis de llanto intenso ${ }^{2}$. Estos episodios ocurren a intervalos de 15 a 20 minutos, haciéndose más frecuentes e intensos con las horas. Además pueden presentar vómitos (biliosos o no) y, si se asocia a deposiciones sanguinolentas tipo "jalea de grosella" con masa abdominal palpable se constituye la tríada clásica del cuadro ${ }^{2,6}$.

Inicialmente, entre los episodios de dolor, el paciente se observa tranquilo, pudiéndose confundir el cuadro con una gastroenteritis, adenitis mesentérica o apendicitis. A medida que progresan los síntomas, se desarrolla un estado de letargia creciente, lo que también plantea como diagnóstico diferencial cuadros de compromiso neurológico o séptico. No obstante, la frecuencia de los síntomas es variable: la tríada clásica sólo se describe en un $15 \%$ a $30 \%$ de los pacientes ${ }^{7}$, mientras que un $10 \%$ de los lactantes pueden no tener signos de dolor evidente ${ }^{7}$.

En lactantes, la mayoría de los casos son idiopáticos ${ }^{7}$, sólo en un 2-8\% de los pacientes se demuestran alteraciones anatómicas que actúan como causantes de la intususcepción ${ }^{4,6}$, siendo la más frecuente el divertículo de Meckel, especialmente en mayores de 2 años y adultos ${ }^{2,4}$.

El divertículo de Meckel es la malformación congénita gastrointestinal más común en la población general, con una incidencia de 1 a $4 \% \%^{5,8-10}$. Su hallazgo es principalmente incidental, pues la mayoría de los portadores se mantienen asintomáticos durante toda la vida. Se estima que los casos sintomáticos corresponden al $1 \%$ del total de portadores ${ }^{10-12}$. Esta alteración anatómica corresponde a un divertículo intestinal verdadero, vestigio embrionario del conducto onfalomesentérico, que normalmente regresa en forma completa entre la quinta y séptima semana de gestación ${ }^{12,13}$. Se ubica generalmente en los 90 centímetros proximales a la válvula ileocecal (hasta 180 centímetros) ${ }^{13}$ y puede presentar en un 50 a $60 \%$ de los casos mucosa heterotópica gástrica, pancreática o hepatobiliar ${ }^{10,12,13}$.

El objetivo de esta comunicación es presentar 2 casos de invaginación ileoileal por divertículo de Meckel, de un total de 20 invaginaciones en el Hospital Regional de Talca ocurridas entre el año 2009 y 2011.

\section{Caso clínico 1}

Paciente de 6 meses de edad, de sexo femenino, sin antecedentes mórbidos. Padres consultan en Servicio de Urgencia por cuadro caracterizado por dolor abdominal cólico de 36 horas de evolución asociado a vómitos y deposiciones sanguinolentas, en dos ocasiones. 
Al ingreso al hospital, la paciente se encontraba afebril, hidratada, eupneica y vigil, resaltando su extrema pasividad, no obstante, con adecuada respuesta a estímulos.

Al examen físico destacaba un abdomen blando, depresible, con sensibilidad difusa en hemiabdomen derecho, sin palpar masas. El resto del examen no mostró hallazgos patológicos.

En base a los antecedentes clínicos se planteó el diagnóstico de invaginación intestinal y se solicitó una radiografía de abdomen simple (al no disponer de una ecografía en forma expedita), en la que se identificaron niveles hidroaéreos generalizados. Este resultado, aunque no certificaba el diagnóstico, sí apoyaba la hipótesis diagnóstica inicial, por lo que se decidió realizar una exploración quirúrgica de urgencia.

Durante el procedimiento se confirmó el diagnóstico de invaginación intestinal, de localización ileoileal. Como primera opción terapéutica se intentó realizar una desinvaginación manual. Mientras se realizaba la maniobra, se halló un divertículo de Meckel a 30 centímetros de la válvula ileocecal, rodeado de áreas cianótico-necróticas, por lo que se completó la desinvaginacion manual y se efectuó una resección de la zona afectada de aproximadamente $8 \mathrm{~cm}$ con posterior anastomosis término-terminal. Finalmente, se realizó apendicectomía profiláctica.

Evolucionó favorablemente, recuperando el tránsito intestinal al segundo día postoperatorio y dándose de alta al séptimo día desde su ingreso.

La paciente en los controles posteriores no presenta complicaciones.

La biopsia confirmó la presencia de una invaginación de tipo diverticular completa con presencia de tejido heterotópico pancreático correspondiente a divertículo de Meckel.

\section{Caso clínico 2}

Paciente de 1 año y 11 meses, de sexo masculino, con antecedentes de Tetralogía de Fallot operada y Síndrome de Di George tratado con Gluconato de Calcio y Vitamina D. Con- sulta por compromiso del estado general y vómitos, lo que se acompaña de deshidratación severa por lo que se hospitaliza y se indica reposición de volumen con solución glucosalina, no encontrándose alteraciones hidroelectrolíticas.

Se mantiene hospitalizado durante 3 días con un cuadro caracterizado por deposiciones semilíquidas no sanguinolentas, hasta que presenta un nuevo episodio de vómitos de carácter bilioso, por lo cual se realizó una radiografía de abdomen que mostró niveles hidroaéreos, sin aire a distal por lo que es derivado al Hospital Regional de Talca.

Es evaluado por cirujano infantil, quien nota al paciente decaído, irritable, con el abdomen muy distendido, poco depresible, con sensibilidad difusa y ruidos hidroaéreos de tono metálico. Se realiza sondeo rectal en el cual no hay salida de gas ni deposiciones, mientras que por sonda nasogástrica se extrae contenido fecaloídeo, diagnosticándose obstrucción intestinal, se indica régimen cero, antibióticos y se prepara para una laparotomía exploratoria.

Se realiza una laparotomía transversa infraumbilical, encontrándose abundante líquido libre claro en la cavidad peritoneal. Las asas intestinales desde el ángulo de Treitz a distal se encontraban dilatadas ubicando una invaginación ileo-ileal a 60 centímetros de la válvula ileocecal. Al desinvaginar se encuentra un divertículo de Meckel que parecía actuar como cabeza de intususcepción. Se resecan aproximadamente 7 centímetros de íleon alrededor del divertículo y se realiza una anastomosis ileoileal término terminal más una apendicectomía.

El paciente evoluciona en forma favorable en el postoperatorio, sin presentar complicaciones.

La biopsia muestra la presencia de una enteritis isquémica asociada a un Divertículo de Meckel.

\section{Discusión}

La invaginación intestinal es la principal causa de obstrucción intestinal en niños menores de 2 años, siendo en este grupo etario 
la mayoría de las veces idiopática. Dentro del grupo de invaginaciones con anomalías anatómicas como causa, se ha identificado al divertículo de Meckel como la más frecuente.

En diversos estudios, el hallazgo de divertículo de Meckel como desencadenante de la intususcepción no supera el 2,8\% de la totalidad de los casos en la edad pediátrica ${ }^{3,4,6,14-16}$, sin embargo, estos datos no son aplicables a lactantes, pues las muestras no están centradas en este grupo y se sabe que en mayores de 2 años las alteraciones anatómicas son más frecuentes, lo que haría sobreestimar la incidencia.

En relación a la presentación clínica de nuestros pacientes, esta difería notablemente entre ellos. El primer caso corresponde a la presentación clásica de invaginación intestinal con dolor abdominal, vómitos y enterorragia; en cambio, el segundo caso no presentó los hallazgos típicos de invaginación, sino que se caracterizó por síntomas de obstrucción a los 3 días de iniciado un cuadro de diarrea no sanguinolenta.

De igual forma, no existen signos clínicos que permitan distinguir una intususcepción idiopática de una causada por una alteración anatómica.

Ante la sospecha de invaginación intestinal se debería realizar una ecografía abdominal, no estando disponible ésta, es útil solicitar una radiografía simple de abdomen como estudio inicial $^{17}$ ya que permite sospechar el cuadro en un porcentaje elevado de los pacientes (60$80 \%)^{6}$, sin embargo, esta prueba no es diagnóstica, generalmente es inespecífica y presenta una cantidad no despreciable de falsos negativos $(20-40 \%)^{6,13}$. Su principal utilidad radica en evaluar la presencia de perforación $u$ obstrucción intestinal ${ }^{18}$, por lo se justificaba su realización en ambos casos. Por último, cabe mencionar que no permite hacer el diagnóstico preoperatorio de un divertículo de Meckel, debido a que no hay signos radiológicos patognomónicos de éste ${ }^{13,15}$.

La ecografía abdominal es en la actualidad el método de diagnóstico radiológico de elección para la intususcepción por su sensibilidad y especificidad, ambas cercanas al $100 \%$ en manos de un operador experimentado ${ }^{6,19}$. Además, permite una mejor detección y caracte- rización de una invaginación en comparación con un enema contrastado, pudiendo detectar una anomalía anatómica como el divertículo de Meckel $^{15}$.

El enema baritado fue el gold estándar para el diagnóstico de intususcepción por muchos años, sin embargo, ha sido desplazado por la ecografía por su mayor eficacia, comodidad y seguridad $^{6,15,18}$. Si bien la tomografía computada es un examen sensible para el diagnóstico de la invaginación intestinal y puede demostrar la presencia de un punto de inicio, no es conveniente usarlo en todos los pacientes pediátricos por el costo, disponibilidad y exposición a radiación que conlleva ${ }^{20}$. La resonancia magnética es más exacta que los métodos ya descritos, pero aún así no tiene un $100 \%$ de rendimiento, lo que asociado al riesgo anestésico y mayor costo, lo que la pone en desventaja frente a la ultrasonografía ${ }^{13}$. Por lo tanto, no se recomiendan estos estudios para la evaluación inicial, menos aún en los casos que hemos descrito.

La realización de una laparotomía exploradora se justifica en los casos presentados pues no se recomienda la reducción hidrostática o neumática cuando hay signos de neumoperitoneo, peritonitis, shock o si existe una alteración anatómica que actúa como cabeza de invaginación, en este caso, un Divertículo de Meckel $^{6,21,22,23}$.

La existencia de tejido pancreático ectópico que presentó el primer caso descrito es infrecuente, encontrándose sólo en el 5 a 15\% de los divertículos de Meckel. No obstante, este hallazgo no tiene significación clínica aparente ${ }^{18}$.

\section{Conclusión}

En la edad pediátrica, en especial en los lactantes, la invaginación intestinal es un cuadro de alta relevancia clínica no sólo por el grado de sospecha que requiere su diagnóstico, sino por las consecuencias que derivan de un manejo inadecuado, presentando una mortalidad global de hasta $6 \%$. En este ámbito, la invaginación intestinal del segmento ileoileal causada por alteraciones anatómicas como el divertículo de Meckel es infrecuente, no tiene 
signos patognomónicos y su estudio no difiere del habitual de la intususcepción. Sin embargo, sí es un factor importante a considerar en el tratamiento, pues tiene indicación quirúrgica y está contraindicada la reducción neumática.

\section{Referencias}

1.- Kolsen T, Bihrmann K, Perch M, et al: Intussusception in Early Childhood: A Cohort Study of 1.7 Million Children. Pediatrics 2004; 114: $782-5$ (http://pediatrics. aappublications.org Última visita el 13-03-2012).

2.- Abate H, Strugo L, Falaschi A: Aspectos clínicos y epidemiológicos de la invaginación intestinal en niños menores de 2 años, de la provincia de Mendoza, Argentina. Arch Argent Pediatr 2006; 104: $496-500$ (http:// www.scielo.org.ar Última visita el 13-03-2012).

3.- Fei-Teng Kong, Wen-Ying Liu, Yun-Man Tang, et al: Intussusception in infants younger than 3 months: a single center's experience. World J Pediatr, 2010; 6: 55-59 (www.springerlink.com Última visita el 13-03-2012).

4.- Le Masne A, Lortat-Jacob S, Sayegh N, Sannier N, Brunelle $F$, Cheron $G$ : Intussusception in infants and children: feasibility of ambulatory management. Eur J Pediatr 1999; 158: 707-10 (www.springerlink.com Última visita el 13-03-2012).

5.- McCollough M, Sharieff G. Abdominal surgical emergencies in infants and Young children. Emerg Med Clin N Am 2003; 21 : 909-35.

6.- Montes P, Soto G, Codoceo A, et al: Enfrentamiento médico quirúrgico de invaginación intestinal. Experiencia de una institución universitaria. Rev Med Chile 2000; 128: 309-14 (www.scielo.cl Última visita el 0901-2013).

7.- Lucero Y, Valenzuela M, O'Ryan M: Perfil epidemiológico y clínico de la invaginación intestinal en lactantes de la Región Metropolitana. Rev Med Chile 2004; 132: 565-72.

8.- Sagar J, Kumar V, Shah D: Meckel's diverticulum: a systematic review. J R Soc Med 2006; 99: 501-5 (http:// jrsm.rsmjournals.com Última visita el 13-03-2012).

9.- Ruscher K, Fisher J, Hughes C, et al: National trends in the surgical management of Meckel's diverticulum. Journal of Pediatric Surgery 2011; 46: 893-6.

10.- Park J, Wolff B, Tollefson M, Walsh E, Larson D: Mec- kel Diverticulum The Mayo Clinic Experience With 1476 Patients (1950-2002). Annals of Surgery 2005; 241: 529-33.

11.- Taek Yu S, Kyun Oh Y, Churl Park W, A Kim E, Woo Lee C, Suk Yoon H: A Case of Intussusception caused by Meckel's Diverticulum in a Newborn. Korean Journal of Pediatrics 2005; 48: 907-10.

12.- Toselli L, Lobos P: Descripción del caso presentado en el número anterior: Divertículo de Meckel. Arch Argent Pediatr 2009; 107: 374-5 (www.scielo.org.ar Última visita el 13-03-2012).

13.- Cardemil F, Gac P, Amat J, et al: Divertículo de Meckel invertido como causa de intususcepción intestinal en el adulto: reporte de un caso. Rev Chilena de Cirugía 2005; 57: 180-3.

14.- Jiménez J: Invaginación intestinal en pediatría. Rev Mex de Cir Pediatría 2005; 12: 195-203.

15.- Shekherdimian S, Lee S: Management of pediatric intussusception in hospitals: diagnosis, treatment, and differences based on age. World J Pediatr 2011; 7: 70-3.

16.- Liu K, MacCarthy J, Guiney E, Fitzgerald R: Intussusception-current trends in management. Arch Dis Childhood 1986; 61: 75-7.

17.- San Román J, Dovasio F, Kreindel T, Kucharczyk M: Invaginación Intestinal. Arch Argent Pediatr 2006; 104: 470-3.

18.- Reijnen J, Festen C, Van Roosmalen R: Intussusception: factors related to treatment, Arch Dis Childhoood 1990; 65: 871-3.

19.- Hryhorczuk AL, Strouse PJ: Validation of US as a firstline diagnostic test for assessment of pediatric ileocolic intussusception. Pediatr Radiol 2009; 39: 1075-9.

20.- Mao-Meng T, Yung-Liang W, Shu-Hang N, et al: Sonographic Features of Small-bowel intussusceptions in Pediatric Patients. Acedemic Emergency Medicine 2001; 8: 368-73.

21.- Del Pozo G, Albillos J, Tejedor D, et al: Intussuscepcion in Children: Current Concepts in Diagnosis and Enema Reduction. RadioGraphics 1999; 19: 299-319.

22.- Cano I: Reducción neumática de invaginación intestinal. Descripción de la técnica y presentación de un caso. Avances 2011; 24-7.

23- Cadavid L, Núñez R, de Barbieri F, Berrios GC, García $B C$ : Reducción neumática de invaginación intestinal en niños: experiencia clínica y técnica. Rev Colomb Radiol 2010; $21: 2827-31$. 